\title{
Intuitionistic fuzzy stability of a quadratic functional equation
}

\author{
Nabin Chandra Kayal ${ }^{1}$, Pratap Mondal ${ }^{2}$, T. K. Samanta ${ }^{3}$ \\ ${ }^{1}$ Department of Mathematics, Moula Netaji Vidyalaya, Moula, Howrah, 711312, West Bengal, India \\ ${ }^{2}$ Department of Mathematics, Orphuli Uday Chand Memorial Institute, Orphuli, Bagnan, Howrah, 711303, West \\ Bengal, India \\ ${ }^{3}$ Department of Mathematics, Uluberia College, Uluberia, Howrah, 711315, West Bengal, India. \\ E-mail: ${ }^{1}$ kayalnabin82@gmail.com, ${ }^{2}$ pratapmondal111@gmail.com, ${ }^{3}$ mumpu_tapas5@yahoo.co.in
}

\begin{abstract}
The aim of this paper is to determine Hyers-Ulam-Rassias Stability results concerning the quadratic functional equation $f(2 x+y)+f(2 x-y)=2 f(x+y)+2 f(x-y)+4 f(x)-2 f(y)$ in intuitionistic fuzzy Banach spaces.
\end{abstract}

2010 Mathematics Subject Classification. 03E72. 97I70,39B82

Keywords. t-norm, t-conorm, Intuitionistic fuzzy normed space, Quadratic functional equation, Hyers-Ulam-Rassias stability.

\section{Introduction}

The study of stability problems for functional equations started with a well-known question raised by Ulam [15] in 1940 regarding the stability of group homomorphisms. In the next year Hyers [3] gave the first affirmative answer to the question of Ulam, for Cauchy functional equation under the assumption that the groups are Banach spaces and this result was generalized by $\mathrm{T}$. Aoki [17] for additive mappings and by Th. M. Rassias [18] for linear mappings by considering an unbounded Cauchy difference. Gavruta [11] generalized Rassias' theorem by replacing the unbounded Cauchy difference by a general control function. F . Skof [4] generalized the Hyers-Ulam stability theorem for the function $f: X \rightarrow Y$, where $X$ is a normed space and $Y$ is a Banach space and then $\mathrm{P}$. W . Cholewa [12] and S. Czerwik [14] extended the result of Skof. Thereafter, several stability problems of various functional equations have been studied and recently fuzzy version is also discussed .

Atanassov [8] introduced the idea of intuitionistic fuzzy sets as a generalization of fuzzy sets . A few notions of intuitionistic fuzzy metric spaces and intuitionistic fuzzy normed spaces were introduced by J. H. Park [7], Saadati and Park [13] and Samanta et.al..[19] .

The stability results of many functional equations have been proved by many researchers $[1$, $2,9,10,16,20,21]$ in fuzzy Banach spaces and intuitionistic fuzzy Banach spaces. Our interest is to established some stability results concerning the quadratic functional equation $f(2 x+y)+$ $f(2 x-y)=2 f(x+y)+2 f(x-y)+4 f(x)-2 f(y)$ in intuitionistic fuzzy Banach spaces .

\section{Preliminaries}

In this section we recall some lemmas, definitions and examples which will be used in this paper.

Lemma 2.1. [6] Consider the set $L^{*}$ and the order relation $\leq_{L^{*}}$ defined by

$$
L^{*}=\left\{\left(x_{1}, x_{2}\right):\left(x_{1}, x_{2}\right) \in[0,1]^{2} \text { and } x_{1}+x_{2} \leq 1\right\},
$$


$\left(x_{1}, x_{2}\right) \leq_{L^{*}}\left(y_{1}, y_{2}\right) \Leftrightarrow x_{1} \leq y_{1}, x_{2} \geq y_{2}, \forall\left(x_{1}, x_{2}\right),\left(y_{1}, y_{2}\right) \in L^{*}$.

Then $\left(L^{*}, \leq_{L^{*}}\right)$ is a complete lattice. We denote its units by $0_{L^{*}}=(0,1)$ and $1_{L^{*}}=(1,0)$.

Definition 2.2. [8] An intuitionistic fuzzy set $A_{\zeta, \eta}$ in a universal set $U$ is an object $A_{\zeta, \eta}=$ $\left\{\left(\zeta_{A}(u), \eta_{A}(u)\right): u \in U\right\}$, where $\zeta_{A}(u) \in[0,1]$ and $\eta_{A}(u) \in[0,1]$ for all $u \in U$ are called the membership degree and the non-membership degree respectively, of $u$ in $A_{\zeta, \eta}$ and furthermore, satisfy $\zeta_{A}(u)+\eta_{A}(u) \leq 1$.

Definition 2.3. [5] A triangular norm ( t-norm ) on $L^{*}$ is a mapping $\tau:\left(L^{*}\right)^{2} \rightarrow L^{*}$ satisfying the following conditions :

(a) $\quad\left(\forall x \in L^{*}\right)\left(\tau\left(x, 1_{L^{*}}\right)=x\right)$ ( boundary condition );

(b) $\quad\left(\forall(x, y) \in\left(L^{*}\right)^{2}\right)(\tau(x, y)=\tau(y, x))$ ( commutativity );

(c) $\quad\left(\forall(x, y, z) \in\left(L^{*}\right)^{3}\right)(\tau(x, \tau(y, z))=\tau(\tau(x, y), z))$ ( associativity );

(d) $\quad\left(\forall\left(x, x^{\prime}, y, y^{\prime}\right) \in\left(L^{*}\right)^{4}\right)\left(x \leq_{L^{*}} x^{\prime}\right.$ and $\left.y \leq_{L^{*}} y^{\prime} \Rightarrow \tau(x, y) \leq_{L^{*}} \tau\left(x^{\prime}, y^{\prime}\right)\right)$ (monotonicity).

A t-norm $\tau$ on $L^{*}$ is said to be continuous if for any $x, y \in L^{*}$ and any sequences $\left\{x_{n}\right\}$ and $\left\{y_{n}\right\}$ which converge to $x$ and $y$ respectively,

$$
\lim _{n \rightarrow \infty} \tau\left(x_{n}, y_{n}\right)=\tau(x, y) .
$$

For example, let $a=\left(a_{1}, a_{2}\right), b=\left(b_{1}, b_{2}\right) \in L^{*}$, consider $\tau(a, b)=\left(a_{1} b_{1}, \min \left\{a_{2}+b_{2}, 1\right\}\right)$ and $M(a, b)=\left(\min \left\{a_{1}, b_{1}\right\}, \max \left\{a_{2}, b_{2}\right\}\right)$.

Then $\tau(a, b)$ and $M(a, b)$ are continuous t-norm.

Now, we define a sequence $\tau^{n}$ recursively by $\tau^{1}=\tau$ and

$$
\tau^{n}\left(x^{(1)}, \cdots, x^{(n+1)}\right)=\tau\left(\tau^{n-1}\left(x^{(1)}, \cdots, x^{(n)}\right), x^{(n+1)}\right)
$$

for all $n \geq 2$ and $x^{(i)} \in L^{*}$.

Definition 2.4. [5] A continuous t-norm $\tau$ on $L^{*}$ is said to be continuous t-representable if there exists a continuous t-norm $*$ and a continuous t-conorm $\diamond$ on $[0,1]$ such that, for all $x=\left(x_{1}, x_{2}\right), y=\left(y_{1}, y_{2}\right) \in L^{*}$,

$$
\tau(x, y)=\left(x_{1} * y_{1}, x_{2} \diamond y_{2}\right) .
$$

Definition 2.5. [5] A negator on $L^{*}$ is any decreasing mapping $N: L^{*} \rightarrow L^{*}$ satisfying $N\left(0_{L^{*}}\right)=1_{L^{*}}$ and $N\left(1_{L^{*}}\right)=0_{L^{*}}$. If $N(N(x))=x$ for all $x \in L^{*}$, then $N$ is called an involutive negator. A negator on $[0,1]$ is a decreasing mapping $N:[0,1] \rightarrow[0,1]$ satisfying $N(0)=1$ and $N(1)=0 . N_{s}$ denotes the standard negator on $[0,1]$ defined by $N_{s}(x)=1-x$ for all $x \in[0,1]$.

Definition 2.6. [16] (1) Let $L=\left(L^{*}, \leq_{L^{*}}\right)$. The triple $(X, P, \tau)$ is said to be an L-fuzzy normed space if $X$ is a vector space, $\tau$ is a continuous t-norm on $L^{*}$ and $P$ is an L-fuzzy set on $X \times(0,+\infty)$ satisfying the following conditions for all $x, y \in X$ and $t, s>0$,

(a) $P(x, t)>0_{L^{*}}$;

(b) $P(x, t)=1_{L^{*}}$ if and only if $x=0$; 
(c) $P(\alpha x, t)=P\left(x, \frac{t}{|\alpha|}\right)$ for all $\alpha \neq 0$;

(d) $P(x+y, t+s) \geq_{L} * \tau(P(x, t), P(y, s))$;

(e) $P(x,):.(0, \infty) \rightarrow L^{*}$ is continuous;

(f) $\lim _{t \rightarrow 0} P(x, t)=0_{L^{*}}$ and $\lim _{t \rightarrow \infty} P(x, t)=1_{L^{*}}$.

In this case $P$ is called an L-fuzzy norm ( briefly, $L^{*}$-fuzzy norm ).

(2) If $P=P_{\mu, \nu}$ is an intuitionistic fuzzy set, then the triple $\left(X, P_{\mu, \nu}, \tau\right)$ is said to be an intuitionistic fuzzy normed space ( briefly, IFN-space). In this case $P=P_{\mu, \nu}$ is called an intuitionistic fuzzy norm on $X$.

Note that, if $P$ is an $L^{*}$-fuzzy norm on $X$, then the following are satisfied :

(i) $P(x, t)$ is nondecreasing with respect to $t$ for all $x \in X$.

(ii) $P(x-y, t)=P(y-x, t)$ for all $x, y \in X$ and $t>0$.

Example 2.7. Let $(X,\|\cdot\|)$ be a normed space.

Let $\tau(a, b)=\left(a_{1} b_{1}, \min \left\{a_{2}+b_{2}, 1\right\}\right)$ for all $a=\left(a_{1}, a_{2}\right), b=\left(b_{1}, b_{2}\right) \in L^{*}$ and $\mu, \nu$ be membership and non-membership degree of an intuitionistic fuzzy set defined by

$$
P_{\mu, \nu}(x, t)=\left(\mu_{x}(t), \nu_{x}(t)\right)=\left(\frac{t}{t+m\|x\|}, \frac{\|x\|}{t+\|x\|}\right)
$$

for all $t \in \mathbb{R}^{+}$in which $m>1$. Then $\left(X, P_{\mu, \nu}, \tau\right)$ is an IFN-space. Here, $\mu(x, t)+\nu(x, t)=1$ for $x=0$ and $\mu(x, t)+\nu(x, t)<1$ for $x \neq 0$.

Let $M(a, b)=\left(\min \left\{a_{1}, b_{1}\right\}, \max \left\{a_{2}, b_{2}\right\}\right)$ for all $a=\left(a_{1}, a_{2}\right), b=\left(b_{1}, b_{2}\right) \in L^{*}$ and $\mu, \nu$ be membership and non-membership degree of an intuitionistic fuzzy set defined by

$$
P_{\mu, \nu}(x, t)=\left(\mu_{x}(t), \nu_{x}(t)\right)=\left(e^{-\frac{\|x\|}{t}}, e^{-\frac{\|x\|}{t}}\left(e^{\frac{\|x\|}{t}}-1\right)\right)
$$

for all $t \in \mathbb{R}^{+}$. Then $\left(X, P_{\mu, \nu}, M\right)$ is an IFN-space.

Definition 2.8. (1) A sequence $\left\{x_{n}\right\}$ in an IFN-space $\left(X, P_{\mu, \nu}, \tau\right)$ is said to be convergent to a point $x \in X$ (denoted by $x_{n} \rightarrow x$ ) if $P_{\mu, \nu}\left(x_{n}-x, t\right) \rightarrow 1_{L^{*}}$ as $n \rightarrow \infty$ for every $t>0$.

(2) A sequence $\left\{x_{n}\right\}$ in an IFN-space $\left(X, P_{\mu, \nu}, \tau\right)$ is said to be a Cauchy sequence if, for any $0<\varepsilon<1$ and $t>0$, there exists $n_{0} \in \mathbb{N}$ such that

$$
P_{\mu, \nu}\left(x_{n}-x_{m}, t\right)>_{L^{*}}\left(N_{s}(\varepsilon), \varepsilon\right)
$$

for all $n, m \geq n_{0}$, where $N_{s}$ is the standard negator.

(3) An IFN-space $\left(X, P_{\mu, \nu}, \tau\right)$ is said to be complete if every Cauchy sequence in $\left(X, P_{\mu, \nu}, \tau\right)$ is convergent in $\left(X, P_{\mu, \nu}, \tau\right)$. A complete intuitionistic fuzzy normed space is called an intuitionistic fuzzy Banach space.

\section{Stability of The Functional Equation}

Throughout this section, we assume that $X, Y, Z$ are real vector spaces.

Theorem 3.1. Let $\psi: X^{2} \rightarrow Z$ be a mapping such that

$$
P_{\mu, \nu}^{\prime}(\psi(2 x, 2 y), t) \geq_{L^{*}} P_{\mu, \nu}^{\prime}(\alpha \psi(x, y), t)
$$


for some $\alpha$ satisfying $0<\alpha<4$ and for all $x, y \in X, t>0$, where $\left(Z, P_{\mu, \nu}^{\prime}, \tau\right)$ is an IFN-space. Let $\left(Y, P_{\mu, \nu}, \tau\right)$ be a complete IFN-space and $f: X \rightarrow Y$ be a mapping such that

$$
\begin{gathered}
P_{\mu, \nu}(f(2 x+y)+f(2 x-y)-2 f(x+y)-2 f(x-y)-4 f(x)+2 f(y), t) \\
\geq{ }_{L^{*}} P_{\mu, \nu}^{\prime}(\psi(x, y), t)
\end{gathered}
$$

for all $x, y \in X$ and $t>0$. Then there exists a unique quadratic mapping $Q: X \rightarrow Y$ such that

$$
P_{\mu, \nu}(f(x)-Q(x), t) \geq_{L^{*}} P_{\mu, \nu}^{\prime}(\psi(x, 0), 2(4-\alpha) t)
$$

and

$$
\frac{f\left(2^{n} x\right)}{4^{n}} \rightarrow Q(x), \text { as } n \rightarrow \infty
$$

for all $x \in X, t>0$.

Proof. Putting $x=y=0$ in $f(2 x+y)+f(2 x-y)=2 f(x+y)+2 f(x-y)+4 f(x)-2 f(y)$, we obtain $f(0)=0$. Again, putting $y=0$ in (3.2), we get for all $x \in X, t>0$,

$$
P_{\mu, \nu}\left(f(x)-4^{-1} f(2 x), t\right) \geq_{L^{*}} P_{\mu, \nu}^{\prime}(\psi(x, 0), 8 t) .
$$

Replacing $x$ by $2^{n} x$ in (3.5) and using (3.1), we have for all $x \in X, t>0, n \in \mathbb{N}$,

$$
P_{\mu, \nu}\left(\frac{f\left(2^{n} x\right)}{4^{n}}-\frac{f\left(2^{n+1} x\right)}{4^{n+1}}, t\right) \geq_{L^{*}} P_{\mu, \nu}^{\prime}\left(\psi(x, 0), 8\left(\frac{4}{\alpha}\right)^{n} t\right) .
$$

Now, for all $x \in X, t>0, n \in \mathbb{N}$ we get

$$
\begin{gathered}
P_{\mu, \nu}\left(f(x)-\frac{f\left(2^{n} x\right)}{4^{n}}, \frac{t}{8} \sum_{i=0}^{n-1}\left(\frac{\alpha}{4}\right)^{i}\right) \\
\geq L_{L^{*}} \tau^{n-1}\left(P_{\mu, \nu}\left(f(x)-\frac{f(2 x)}{4}, \frac{t}{8}\right), P_{\mu, \nu}\left(\frac{f(2 x)}{4}-\frac{f\left(2^{2} x\right)}{4^{2}}, \frac{t}{8} \frac{\alpha}{4}\right),\right. \\
\left.\cdots, P_{\mu, \nu}\left(\frac{f\left(2^{n-1} x\right)}{4^{n-1}}-\frac{f\left(2^{n} x\right)}{4^{n}}, \frac{t}{8}\left(\frac{\alpha}{4}\right)^{n-1}\right)\right) \\
\geq L_{L^{*}} P_{\mu, \nu}^{\prime}(\psi(x, 0), t) \quad[\text { by }(3.5),(3.6)] .
\end{gathered}
$$

It implies that for all $x \in X, t>0$ and $n \in \mathbb{N}$,

$$
P_{\mu, \nu}\left(f(x)-\frac{f\left(2^{n} x\right)}{4^{n}}, t\right) \geq_{L^{*}} P_{\mu, \nu}^{\prime}\left(\psi(x, 0), \frac{8 t}{\sum_{i=0}^{n-1}\left(\frac{\alpha}{4}\right)^{i}}\right)
$$


Replacing $x$ by $2^{p} x$ in (3.7) and using (3.1), we get for all $x \in X, t>0$ and $n, p \in \mathbb{N}$,

$$
P_{\mu, \nu}\left(\frac{f\left(2^{p} x\right)}{4^{p}}-\frac{f\left(2^{n+p} x\right)}{4^{n+p}}, t\right) \geq_{L^{*}} P_{\mu, \nu}^{\prime}\left(\psi(x, 0), \frac{8 t}{\left(\frac{\alpha}{4}\right)^{p} \sum_{i=0}^{n-1}\left(\frac{\alpha}{4}\right)^{i}}\right) .
$$

Taking limit as $p \rightarrow \infty$, we get for all $x \in X, n \in \mathbb{N}$ and $t>0$,

$$
P_{\mu, \nu}\left(\frac{f\left(2^{p} x\right)}{4^{p}}-\frac{f\left(2^{n+p} x\right)}{4^{n+p}}, t\right) \rightarrow 1_{L^{*}} \text { as } p \rightarrow \infty .
$$

Therefore the sequence $\left\{\frac{f\left(2^{n} x\right)}{4^{n}}\right\}$ is a Cauchy sequence in $\left(Y, P_{\mu, \nu}, \tau\right)$. Since $\left(Y, P_{\mu, \nu}, \tau\right)$ is a complete IFN-space, there exists some function $Q: X \rightarrow Y$ such that (3.4) holds. Let $\delta>0$. Now, for all $x \in X, t>0$ and $n \in \mathbb{N}$,

$$
\begin{gathered}
P_{\mu, \nu}(f(x)-Q(x), t+\delta) \\
\geq_{L^{*} \tau}\left(P_{\mu, \nu}^{\prime}\left(\psi(x, 0), \frac{8 t}{\sum_{i=0}^{n-1}\left(\frac{\alpha}{4}\right)^{i}}\right), P_{\mu, \nu}\left(\frac{f\left(2^{n} x\right)}{4^{n}}-Q(x), \delta\right)\right) \quad[\text { by }(3.7)] .
\end{gathered}
$$

Taking limit as $n \rightarrow \infty$, we get for all $x \in X, t>0$,

$$
P_{\mu, \nu}(f(x)-Q(x), t+\delta) \geq_{L^{*}} \tau\left(P_{\mu, \nu}^{\prime}\left(\psi(x, 0), \frac{8 t}{\sum_{i=0}^{\infty}\left(\frac{\alpha}{4}\right)^{i}}\right), 1_{L^{*}}\right) \quad[\text { by (3.4)]. }
$$

Letting $\delta \rightarrow 0$, we get (3.3). From definition of $Q$ we get for all $x \in X$ and $n \in \mathbb{N}$,

$$
Q\left(2^{n} x\right)=4^{n} Q(x) .
$$

We replace $x$ and $y$ by $2^{n} x$ and $2^{n} y$ respectively in (3.2) to get for all $x, y \in X, t>0$ and $n \in \mathbb{N}$,

$$
\begin{gathered}
P_{\mu, \nu}\left(\frac{f\left(2^{n}(2 x+y)\right)}{4^{n}}+\frac{f\left(2^{n}(2 x-y)\right)}{4^{n}}-\frac{2 f\left(2^{n}(x+y)\right)}{4^{n}}\right. \\
\quad-\frac{2 f\left(2^{n}(x-y)\right)}{4^{n}}-\frac{4 f\left(2^{n} x\right)}{4^{n}}+\frac{2 f\left(2^{n} y\right)}{\left.4^{n}, t\right)} \\
\geq{ }_{L^{*}} P_{\mu, \nu}^{\prime}\left(\psi\left(2^{n} x, 2^{n} y\right), 4^{n} t\right) \\
\geq{ }_{L^{*}} P_{\mu, \nu}^{\prime}\left(\psi(x, y),\left(\frac{4}{\alpha}\right)^{n} t\right) \quad[\text { by }(3.1)] .
\end{gathered}
$$


Taking limit $n \rightarrow \infty$, we get for all $x, y \in X, t>0$,

$$
P_{\mu, \nu}(Q(2 x+y)+Q(2 x-y)-2 Q(x+y)-2 Q(x-y)-4 Q(x)+2 Q(y), t)=1_{L^{*}} .
$$

It implies that for all $x, y \in X$,

$$
Q(2 x+y)+Q(2 x-y)-2 Q(x+y)-2 Q(x-y)-4 Q(x)+2 Q(y)=0 .
$$

This shows that $Q$ is quadratic. To prove the uniqueness we assume that $Q^{\prime}: X \rightarrow Y$ is a quadratic function satisfying (3.3) and (3.4). Now, using (3.9), (3.3) and (3.1), we get for all $x \in X, t>0$ and $n \in \mathbb{N}$,

$$
\begin{gathered}
P_{\mu, \nu}\left(Q(x)-Q^{\prime}(x), t\right) \\
\geq_{L^{*}} \tau\left(P_{\mu, \nu}\left(f\left(2^{n} x\right)-Q\left(2^{n} x\right), \frac{4^{n} t}{2}\right), P_{\mu, \nu}\left(f\left(2^{n} x\right)-Q^{\prime}\left(2^{n} x\right), \frac{4^{n} t}{2}\right)\right) \\
\geq_{L^{*}} \tau\left(P_{\mu, \nu}^{\prime}\left(\psi(x, 0), \frac{4^{n}(4-\alpha) t}{\alpha^{n}}\right), P_{\mu, \nu}^{\prime}\left(\psi(x, 0), \frac{4^{n}(4-\alpha) t}{\alpha^{n}}\right)\right) .
\end{gathered}
$$

Taking limit as $n \rightarrow \infty$, we get for all $x \in X, t>0$,

$$
P_{\mu, \nu}\left(Q(x)-Q^{\prime}(x), t\right)=1_{L^{*}}
$$

It implies that $Q(x)=Q^{\prime}(x)$ for all $x \in X$. This proves that $Q$ is unique.

This completes the proof.

Q.E.D.

Corollary 3.2. Let $\left(Y, P_{\mu, \nu}, \tau\right)$ be a complete IFN-space and $\left(Z, P_{\mu, \nu}^{\prime}, \tau\right)$ be an IFN-space. Let $p, q$ be two non-negative real numbers less than 1 and $z_{0} \in Z$. Let $f: X \rightarrow Y$ be a mapping such that

$$
\begin{gathered}
P_{\mu, \nu}(f(2 x+y)+f(2 x-y)-2 f(x+y)-2 f(x-y)-4 f(x)+2 f(y), t) \\
\geq_{L^{*}} P_{\mu, \nu}^{\prime}\left(\left(\|x\|^{p}+\|y\|^{q}\right) z_{0}, t\right)
\end{gathered}
$$

for all $x, y \in X$ and $t>0$. Then there exists a unique quadratic mapping $Q: X \rightarrow Y$ such that

$$
P_{\mu, \nu}(f(x)-Q(x), t) \geq_{L^{*}} P_{\mu, \nu}^{\prime}\left(\|x\|^{p} z_{0}, 2\left(4-4^{p}\right) t\right)
$$

and

$$
\frac{f\left(2^{n} x\right)}{4^{n}} \rightarrow Q(x), \text { as } n \rightarrow \infty
$$

for all $x \in X, t>0$.

Proof. Define $\psi(x, y)=\left(\|x\|^{p}+\|y\|^{q}\right) z_{0}$ and take $\alpha=4^{p}$. Clearly, (3.1) is satisfied and $0<\alpha<4$. Thus the theorem (3.1) completes the proof. 
Corollary 3.3. Let $\left(Y, P_{\mu, \nu}, \tau\right)$ be a complete IFN-space and $\left(Z, P_{\mu_{1}, \nu_{1}}^{\prime}, \tau\right)$ be an IFN-space. Let $z_{0} \in Z, \varepsilon \geq 0$ and $f: X \rightarrow Y$ be a mapping such that

$$
\begin{aligned}
P_{\mu, \nu}(f(2 x+y)+f(2 x-y) & -2 f(x+y)-2 f(x-y)-4 f(x)+2 f(y), t) \\
& \geq_{L^{*}} P_{\mu, \nu}^{\prime}\left(\varepsilon z_{0}, t\right)
\end{aligned}
$$

for all $x, y \in X$ and $t>0$. Then there exists a unique quadratic mapping $Q: X \rightarrow Y$ such that

$$
P_{\mu, \nu}(f(x)-Q(x), t) \geq_{L^{*}} P_{\mu, \nu}^{\prime}\left(\varepsilon z_{0}, t\right)
$$

and

$$
\frac{f\left(2^{n} x\right)}{4^{n}} \rightarrow Q(x) \text {, as } n \rightarrow \infty
$$

for all $x \in X, t>0$.

Proof. Define $\psi(x, y)=\varepsilon z_{0}$ and take $\alpha=3.5$. Clearly, (3.1) is satisfied. Thus the theorem (3.1) completes the proof.

Theorem 3.4. Let $\psi: X^{2} \rightarrow Z$ be a mapping such that

$$
P_{\mu, \nu}^{\prime}\left(\psi\left(\frac{x}{2}, \frac{y}{2}\right), t\right) \geq_{L^{*}} P_{\mu, \nu}^{\prime}\left(\frac{1}{\alpha} \psi(x, y), t\right)
$$

for some $\alpha>4$ and for all $x, y \in X, t>0$, where $\left(Z, P_{\mu, \nu}^{\prime}, \tau\right)$ is an IFN-space. Let $\left(Y, P_{\mu, \nu}, \tau\right)$ be a complete IFN-space and $f: X \rightarrow Y$ be a mapping such that

$$
\begin{gathered}
P_{\mu, \nu}(f(2 x+y)+f(2 x-y)-2 f(x+y)-2 f(x-y)-4 f(x)+2 f(y), t) \\
\geq{ }_{L^{*}} P_{\mu, \nu}^{\prime}(\psi(x, y), t)
\end{gathered}
$$

for all $x, y \in X$ and $t>0$. Then there exists a unique quadratic mapping $Q: X \rightarrow Y$ such that

$$
P_{\mu, \nu}(f(x)-Q(x), t) \geq_{L^{*}} P_{\mu, \nu}^{\prime}(\psi(x, 0), 2(\alpha-4) t)
$$

and

$$
4^{n} f\left(\frac{x}{2^{n}}\right) \rightarrow Q(x), \text { as } n \rightarrow \infty
$$

for all $x \in X, t>0$.

\section{References}

[1] A. K. Mirmostafaee, M. S. Moslehian, Fuzzy versions of Hyers-Ulam-Rassias theorem, Fuzzy Sets and Systems, 159 (2008), 720729. 
[2] C. Park, Fuzzy stability of a functional equation associated with inner product space, Fuzzy Sets and Systems, 160 (2009), 16321642.

[3] D. H. Hyers, On the stability of the linear functional equation, Proc. Nat. Acad. Sci. U.S.A., 27 (1941), 222-224.

[4] F. Skof, Proprieta locali e approssimazione di opratori, Rend. Sem. Mat. Fis. Milano, 53 (1983), $113-129$.

[5] G. Deschrijver, C. Cornelis and E. E. Kerre, On the representation of intuitionistic fuzzy tnorms and t-conorms, IEEE Transaction on Fuzzy Systems, 12 (2004), 45-61.

[6] G. Deschrijver, E. E. Kerre, On the relationship between some extensions of fuzzy set theory, Fuzzy Sets and Systems, 23 (2003), 227-235.

[7] J. H. Park, Intuitionistic fuzzy metric spaces, Chaos, Solitons and Fractals, 22 (2004), $1039-1046$.

[8] K. T. Atanassov, Intuitionistic fuzzy sets, Fuzzy Sets and Systems, 20 (1986), 87-96.

[9] N. Chandra Kayal, P. Mondal and T. K. Samanta The Generalized Hyers - Ulam - Rassias Stability of a Quadratic Functional Equation in Fuzzy Banach Spaces, Journal of New Results in Science, 1 (5) (2014), 83-95.

[10] N. Chandra Kayal, P. Mondal and T. K. Samanta, The Fuzzy Stability of a Pexiderized Functional Equation, Mathematica Moravica, 18 (2) (2014), 1-14.

[11] P. Gavruta, A generalization of the Hyers-Ulam-Rassias stability of approximately additive mappings, J. Math. Anal. appl., 184 (1994), 431-436.

[12] P. W. Cholewa, Remarks on the stability of functional equations, Aequationes Math., 27 (1984), $76-86$.

[13] R. Saadati, J. H. Park, On the intuitionistic fuzzy topological spaces, Chaos, Solitons and Fractals, 27 (2006), 331-344.

[14] S. Czerwik, On the stability of the quadratic mappings in normed spaces, Abh. Math. Sem. Univ. Hamburg, 62 (1992), 59-64.

[15] S. M. Ulam, Problems in Modern Mathematics, Chapter VI, Science Editions, Wiley, New York, 1960.

[16] S. Shakeri, Intutionistic fuzzy stability of Jenson type mapping, J. Non linear Sc. Appl., 2 (2009), no.-2, 105-112.

[17] T. Aoki, On the Stability of Linear Transformation in Banach Spaces, J. Math. Soc. Japan, 2 (1950), 64-66.

[18] Th. M. Rassias, On the stability of the linear mapping in Banach space, Proc. Amer. Mathematical Society, 72(2) (1978), 297-300. 
[19] T. K. Samanta and Iqbal H. Jebril, Finite dimentional intuitionistic fuzzy normed linear space, Int. J. Open Problems Compt. Math., 2( 4) (2009), 574591.

[20] T. K. Samanta, P. Mondal and N. Chandra Kayal, The generalized Hyers-Ulam-Rassias stability of a quadratic functional equation in fuzzy Banach spaces, Annals of Fuzzy Mathematics and Informatics, Vol. 6, No. 2, (2013), 285294.

[21] T. K. Samanta, N. Chandra Kayal and P. Mondal, The Stability of a General Quadratic Functional Equation in Fuzzy Banach Space, Journal of Hyperstructures, 1 (2) (2012), 71-87. 